\title{
Discussion on Financial Budget Management System of Modern Logistics Enterprises
}

\author{
Ning Bai \\ Xinhua College of Ningxia University, Xinhua, Ningxia, 750021
}

Keywords: Modern Logistics Enterprises, Financial Budget, Management System, Countermeasures.

\begin{abstract}
The logistics budget management system of modern logistics enterprises is of great significance to realize the strategic goal of logistics enterprises. This article first elaborated the logistics enterprise financial budget management problems, after these problems, analyzed the specific solution.
\end{abstract}

\section{Introduction}

The last century, 20 years, the United States General Electric, DuPont and General Motors and other companies began to use a comprehensive budget management model and the management model quickly became the United States large industrial and commercial enterprises standard operating standards. After more than eighty years of time, the overall budget management has gradually become a very rich gold management practices, in the management of many large enterprises in the United States occupies a very important position. Later, many large and medium-sized enterprises in China gradually found the important role of comprehensive budget management a survey shows that at this stage of our country into the world's top five hundred enterprises in the use of a comprehensive budget management, half of large and medium-sized state-owned enterprises All of the above companies have used full budget management. This shows that budget management is a very critical component of modern enterprise management.

The Ministry of Finance issued on the financial budget management guidance pointed out that the enterprise's financial budget is in the forecast and decision-making conditions, according to corporate strategic objectives to plan and arrange a period of time within the enterprise income and expenditure, business Results and their distribution. The key content of logistics enterprise budget management is its financial budget management and its budget is actually a quantitative financial planning, covering the operating budget, capital budget and financial budget three aspects. The budget is used to constrain the capital investment and financing plan of the enterprise. The financial budget is used to constrain the cash flow quantitative index under the operating budget and capital budget. The

Logistics enterprise financial budget management has the following characteristics: First, the strategic guidance. With the specific strategic planning, logistics companies will have a rule to follow the financial budget. Second, binding. The compilation, execution and control of the financial budget of the logistics enterprise should be carried out according to certain specifications to ensure that the various budget links can be coordinated and connected. Third, with a certain degree of rigidity and flexibility. Rigidity refers to the mandatory and authoritative nature of the budget system, in order to ensure that the budget can be implemented in place, flexibility is to make the budget more feasible and real-time correction rules. Fourth, involving a wide range. Logistics enterprise budget management involves all employees within the enterprise, and related to all aspects of the logistics process, each employee or the link will have a problem on the strategic objectives of the budget impact, it should try the overall management of the budget, To a variety of factors that affect the financial performance of enterprises reasonable control. 


\section{The Logistics Business Financial Budget Management Problems Encountered}

Strategic budget and tactical budgets form the financial budget system of logistics enterprises. After a lot of tactical budget is completed, it is possible to complete the overall goal of the strategic budget, in the strategic financial budget layout, the tactical financial budget can be planned and launched in an orderly manner. First, the strategic financial budget and tactical financial budget has a different focus. The ultimate goal of strategic budget control is as important as the strategic objective of the logistics enterprise to show the level of operation and the allocation of budget resources for a long period of time. The specific budget of the budget project is based on the estimated amount of the estimated revenue to determine. Tactical financial budget is more focused on a short period of time the logistics enterprises in order to achieve the objectives and the need for financial resources control. Many logistics companies can not look long-term, in order to be able to obtain performance in the short term, put all the budget resources into the fast return of the project, for those who later enhance the competitiveness of the enterprise's strategic budget resources investment turn a blind eye, So that there is only a conflict between the holder of the current interest and the holder of the long-term interest. The main reason for this conflict is that there is no reasonable communication and communication between the decision makers of the corporate budget and the budget stakeholders, and the different departments have adopted a method for the overall development of the enterprise for their own interests. Of the budget system are in accordance with the interests of the sector to maximize the idea to carry out, did not stand on the overall development of the enterprise point of view to think and analyze the problem. Second, the logistics enterprises in a long period of time after the financial operation experience will sum up a set of scientific laws of financial operation in order to develop a strategic financial budget based on the relevant decision-making. And tactical financial budget only need to follow the schedule of financial operation can make decisions. Because some of the asset logistics companies will invest a lot of assets in the early stage, so the asset investment cycle can not be consistent with the investment income cycle frequency, if only standing in the short-term financial budget point of view to analyze the longer period of investment projects may be feasible The Therefore, the logistics enterprises should be based on the overall analysis of the problem, the strategic budget and tactical budget cash flow coordination, in order to make the enterprise short-term capital resource needs and long-term capital resource demand scheduling can be carried out in an orderly manner.

In the past, the logistics enterprises will generally refer to the relevant data of the pre-business balance sheet, the budget and the appropriation of the cash flow statement, the profit and loss account and the relevant data forecast in the current period, given the fixed financial budget in the next time period. This traditional approach is based on a certain period of time to weave the budget, the budget resolution is fixed, need to be implemented in the latter part of the process can not be changed, it has become a static budget management. This static budget management model for the business volume is not a small change in the logistics business is more appropriate, and more logistics enterprises because the business has been marketed, so its service prices and costs will change with the market changes, if In accordance with the static budget method to carry out, then there will be the amount of budget than the actual amount of the phenomenon of lag, and because the static budget in the process of environmental changes in the corresponding changes in the ability to complete the specific business assessment and performance evaluation, so there is no The meaning of the budget.

In the past, the budget management method is more focused on the management of the amount of expenditure of the project capital cost. On the basis of determining the project logistics service ability, the expenditure of the logistics service cost is reduced. This analysis mode is static view of the capital value, ignoring the budget funds the value of the time taken. The time value of the funds refers to the value of the capital in the production and circulation over time, so the difference in the expenditure time of the budget funds within a fixed time determines the difference in the value of the present time. If the time value of the funds is neglected in determining the budget plan, it will make the funding structure of the enterprise a lack of balanced arrangements, the cost of capital is 
greatly improved, but also make the use of funds significantly reduced efficiency, the enterprise is extremely limited resources Wasted, making the company face greater financial risk. Because the capital itself will not increase because of the passage of time, it should be through the investment project to increase revenue. If you want to find the balance between budget management and the time value of the funds, you should greatly increase the management capacity of the budget funds, improve the accuracy of the budget management time of the budget funds, and maximize the idle time of the funds Reduce, in order to increase the time value of corporate monetary funds. It can be solved by modifying the method of fund raising and adjusting the time of fund acquisition and return.

\section{The Countermeasures in Logistics Business Financial Budget Management}

The essence of the steps to formulate the financial budget of the logistics enterprise is the process of the redistribution of the internal resources of the enterprise, which is related to the different departments and all the employees' interests in the enterprise. Therefore, the relevant budget management system should be regulated. Because many logistics companies do not have a reasonable scientific and budget management standards as a reference, so there will be job responsibilities are not clear, lack of work process, can not implement specific programs and other issues, if you want to let these problems get a better solution, Should be carried out from the following parts: First, the logistics enterprises in accordance with their own specific circumstances to develop budget management practices, the financial budget management processes and systems clear down. Secondly, the responsibility of the relevant system clause in the budget management specification is implemented to the people, and the time node is defined for each budget, and quantitative indicators are given to better supervise and manage to prevent the definition of responsibility from becoming unclear, Duties responsible person blurred and so on. Finally, within the logistics enterprises to strengthen financial control capabilities, the financial budget subjects to refine, through this approach to improve the overall quality of the budget program, in order to more in line with the logistics business environment. The budget plan should refine the budget expenditure subject, avoid the implementation of the understanding of the deviation, the executive in the implementation of the discretion to minimize the right to control corruption.

The basis of financial budget management of logistics enterprises is to scientifically and rationally forecast the infrastructure investment of logistics facilities and equipment of enterprises, and reasonably forecast the expenses of logistics activities such as storage, transportation and transportation, packaging and so on. First, the logistics company's financial budget program is the overall structure of the logistics business through the strategic objectives of the decision. The operation of modern logistics involves different regions and different industries, so the enterprise logistics management activities, logistics functions, coordination between the functions of the work should be based on the strategic plan as a standard. The strategic objectives of the logistics enterprises can be divided into many small targets, and the coordination between the small targets makes the operational performance of the logistics enterprises be optimized. An important part of the strategic objectives of an enterprise is the financial budget, so it should interact with the rest of the alphabet to make it possible for the logistics company to achieve its strategic objectives. Second, whether the financial budget plan reasonably determines the progress of the strategic objectives of the logistics enterprises. Logistics enterprises can be divided into a lot of strategic goals, these small goals gradually realized, from the overall strategic objectives further, and these small goals to achieve the results and progress is also determined by the financial budget. Scientific arrangements and the development of logistics enterprises strategic objectives have the various small targets to be reasonably arranged to effectively control the progress of corporate strategic objectives, so that the implementation process more optimized.

Logistics management of the enterprise is the need for all employees involved in the enterprise, at the same time in all stages of business management should be implemented. It covers all kinds of management practices such as planning, organization, control, incentive and evaluation. The key to the control of the operational risk and financial risk in the logistics enterprise lies in the financial 
budget management. The concrete and comprehensive implementation of the financial budget management is conducive to consolidating the work of the budget supervision system and improving the financial budget risk control of the enterprise effectively. The financial budget management of logistics enterprises can be strengthened from the following parts: First, coordinate the interests of various departments within the logistics enterprises, strengthen the communication and communication between various departments to identify the risks that affect the healthy development of enterprises factor. Second, in accordance with the designated program, implementation, assessment, evaluation and other steps to consider each of the financial goals, as a standard in terms of rational allocation of financial resources, making the enterprise resources can be optimized. Third, all employees of the logistics enterprise are encouraged to join the financial budget management as a standard to control the various stages of the formulation and implementation of the financial budget. The whole process control for budget management should be controlled in advance, in and afterwards. Prior to the actual situation to develop a feasibility plan; always strengthen the control of the implementation of the budget, encounter any problems should be in the shortest possible time to find a solution; after the implementation of the budget to assess the situation, compared to the budget and the actual The difference between the number of different ways to deal with differences. Execution and feedback are the most important part of the overall budget management. If the implementation is not in place and can not be controlled reasonably, the whole budgeting result will be affected, so that the budget target of the logistics enterprise can not be reached quickly. The process of budget execution is related to the various stages of enterprise operation and management, but also involves a lot of employees, so if you want to ensure the stability of the budget can be implemented, it should encourage all employees to join the budget management, to ensure the general direction of budget management No errors on the basis of strengthening the management of the various sub-budget.

\section{Conclusion}

Based on the above analysis, logistics enterprises should adjust the relevant rules of financial budget management, according to the objectives of logistics enterprises to scientific and rational arrangements for financial budget program, while strengthening the logistics business financial budget management capacity. Only in this way, it is possible to better financial budget management, and ultimately achieve the strategic objectives of logistics enterprises.

\section{Acknowledgements}

Ningxia University Xinhua School Logistics Management Key professional group construction project

\section{References}

[1] Zhang Pingxiang. The application of money time value in investment decision-making [J]. Economy and Management, 2012, 26 (2): 35-38.

[2] Water plum, An Xianglin, Shang Yawen. Problems and countermeasures of financial management in logistics enterprises [J]. Logistics Science and Technology, 2012 (2): 70-72.

[3] Duan Lina. Dynamic budget in the enterprise application problems and countermeasures [J]. Economic Research Guide, 2011 (28): 24-25.

[4] Liu Xiuqin, Chen Linhua.Design of Logistics Cost Financial Budget System for Manufacturing Enterprises [J]. Logistics Technology, 2011, 30 (11): 194-195. 